Article

\title{
Zr-Based MOF-808 as Meerwein-Ponndorf-Verley Reduction Catalyst for Challenging Carbonyl Compounds
}

\author{
Eva Plessers, Guangxia Fu, Collin Yong Xiang Tan, Dirk E. De Vos and Maarten B. J. Roeffaers * \\ Centre for Surface Chemistry and Catalysis, KU Leuven, Celestijnenlaan 200F, 3001 Heverlee, Belgium; \\ eva.plessers@kuleuven.be (E.P.); guangxia.fu@kuleuven.be (G.F.); collin.tan@kuleuven.be (C.Y.X.T.); \\ dirk.devos@kuleuven.be (D.E.D.V.) \\ * Correspondence: maarten.roeffaers@kuleuven.be; Tel.: +32-16-327-449
}

Academic Editor: Minhua Shao

Received: 13 June 2016; Accepted: 13 July 2016; Published: 19 July 2016

\begin{abstract}
In the fine chemical industry, transfer hydrogenation of carbonyl compounds is an important route to selectively form the corresponding allyl alcohol. The Meerwein-Ponndorf-Verley reduction (MPV) is catalyzed by a Lewis acid catalyst and easily oxidizable alcohols serve as hydrogen donor. We successfully used the Zr-based metal-organic framework (MOF) MOF-808-P as MPV-catalyst with isopropyl alcohol as solvent and hydride donor. After only $2 \mathrm{~h}, 99 \%$ yield of cinnamyl alcohol was obtained. The highly active MOF-808-P is also a good catalyst for the selective reduction of more challenging substrates such as $R$-carvone and $\beta$-ionone. Two strategies were successfully used to shift the equilibrium towards the desired allylic alcohol products: (1) evaporation of formed acetone and (2) the use of the more strongly reducing 1-indanol. Carveol yield was increased to $>70 \%$. These results highlight the great potential of this recently discovered Zr-MOF as a chemically and thermally stable catalyst.
\end{abstract}

Keywords: chemoselective catalytic reduction; Meerwein-Ponndorf-Verley; metal-organic framework; heterogeneous Lewis acid; unsaturated aldehyde and ketone; MOF-808

\section{Introduction}

In the fragrance and pharmaceutical industry, allylic alcohols are important intermediates and flavoring compounds [1]. A widespread route to obtaining these allylic alcohols is via the chemoselective reduction of unsaturated aldehydes and ketones. This reduction can be performed via hydrogen transfer from easily oxidizable alcohols catalyzed by a Lewis acid or basic catalyst, a procedure called Meerwein-Ponndorf-Verley reduction (MPV) [2]. MPV reductions are typically performed under mild conditions and with high chemoselectivity because of the low risk of reducing other functional groups or unsaturated $\mathrm{C}=\mathrm{C}$-bonds. The high selectivity can be explained by the mechanism in which a cyclic six-membered transition state is formed [3,4]. Hydrogen donor and acceptor coordinate simultaneously to the Lewis acidic metal center; via this coordination, the carbonyl group of the substrate is activated for the hydride transfer and the reduction is performed with high selectivity [5]. Isopropyl alcohol (IPA) is the most widely used hydride donor because it is easily oxidized to acetone and is environmentally friendly [6]. The major disadvantage of IPA, however, is the reversibility of the reaction [7]. The reverse complementary oxidation of alcohols is known as the Oppenauer oxidation, resulting in an equilibrium called the Meerwein-Ponndorf-Verley-Oppenauer (MPVO) redox equilibrium [5]. To direct the equilibrium towards the desired allylic alcohol, IPA is often used in excess as the solvent of the reaction.

The earliest reported MPV-catalysts were aluminum alkoxides [8]; recent improvements of these homogeneous aluminum catalysts lead to the development of highly efficient aluminum 
siloxide [9] and enantioselective calixarene-based MPV-catalysts [10,11]. However, in the last 15 years, zirconium-based catalysts are gaining interest [3,7,12-15]. The earliest reported Zr-catalysts were unsupported zirconia. In the MPVO reaction of ethanol and acetone, the weakly basic $\mathrm{ZrO}_{2}$ performed less well than the strongly basic $\mathrm{MgO}$ [16]. Hydrous zirconia, on the other hand, proved to be a good MPV-catalyst, giving a 78\% cinnamyl alcohol yield [3], and could be used under flow conditions [17]. Grafting of Zr on siliceous SBA-15 (SBA for Santa Barbara Amorphous type material) or zeolite Beta dramatically decreased the necessary $\mathrm{Zr}$ /substrate ratio [18-20]. The Zr-zeolite Beta catalyst - with $\mathrm{Zr}$ substituted isomorphously into the lattice - was reusable, stable in water, and only $1.3 \mathrm{~mol} \% \mathrm{Zr}$ was used to reach $96 \%$ cinnamyl alcohol yield within reasonable reaction time. Lewis acidity of the Zr-atoms in zeolite Beta was increased by a two-step post-synthesis method to reach $100 \%$ cinnamyl alcohol yield [21].

Cinnamaldehyde is one of the most widely used $\alpha, \beta$-unsaturated aldehydes. It is an attractive MPV-substrate since the unsaturated alcohol product is used as a flowery note [3], and as raw material in the fragrance and pharmaceutical industry-e.g., for the cardiovascular drug cinnarizine, which is derived from cinnamyl alcohol [22]. Another reason for studying cinnamaldehyde as a reactant is the fact that it is relatively easily reduced chemoselectively compared to other unsaturated aldehydes or ketones because of favorable steric and electronic properties. Citral is another widely studied MPV-substrate for which typically lower yields are reached, so longer reaction time or higher Zr-loadings are necessary $[15,19,21,23]$. Carvone, isophorone, and $\beta$-ionone are examples of $\alpha, \beta$-unsaturated ketones that are even more difficult to chemoselectively reduce to obtain allylic alcohols in high yields. De bruyn, et al. [7,24], however, overcame this limitation by using the more strongly reducing 1-indanol as hydrogen donor and siliceous MCM-41 (MCM for Mobil Crystalline Material) immobilized $\mathrm{Zr}$ and $\mathrm{Hf}$ as catalysts.

Aside from zeolites and mesoporous silicas, Zr-containing metal-organic frameworks (MOFs) have recently been proposed as MPV-catalysts. MOFs are crystalline, porous materials composed of metal (oxide) nodes interlinked by polytopic organic ligands, forming three-dimensional structures with well-defined cages and channels. These microporous solids have attracted researchers' interest mainly due to their versatility, high surface area, and acid-base properties. Even though concerns have been raised about their thermal and physical stability, MOFs are promising catalysts for the synthesis of high-added-value products [25-27]. UiO-66 ( $\mathrm{UiO}$ for University of Oslo) is a zirconium-terephthalate-based MOF best known for its high chemical and thermal stability and easy functionalization. When $\mathrm{HCl}$ and/or trifluoroacetic acid are added to the MOF synthesis mixture, terephthalate linkers are partially replaced by trifluoroacetate, resulting in a more open framework with a large number of open sites [14]. These $\mathrm{Zr}$-based modulated $\mathrm{UiO}-66$ and $\mathrm{UiO}-66-\mathrm{NO}_{2}$ were already successfully used as MPV reduction catalysts with tert-butylcyclohexanone as the reactant [14] and in other challenging selective reductions, such as those of unsaturated aldehydes and ketones [28]. Llabres and co-workers [29] successfully showed the presence of Lewis acid centers in UiO-66 and used these Zr-based MOFs for the esterification of levulinic acid with various alcohols. The catalytically-active sites are coordination vacancies on the Zr-metal, arising from crystalline defects associated to linker deficiencies or from thermal dehydroxylation of the $\mathrm{Zr}$-cluster [29,30].

Recently, a new range of $\mathrm{Zr}-\mathrm{MOF}$ has been reported, all based on the $\mathrm{Zr}_{6} \mathrm{O}_{4}(\mathrm{OH})_{4}\left(-\mathrm{CO}_{2}\right)_{n}$ secondary building unit (SBU) and variously shaped carboxylic organic linkers [31]. One of these $\mathrm{Zr}$-materials is MOF-808, in which the Zr-SBU is connected to six 1,3,5-benzenetricarboxylate (BTC) organic linkers and six formate ligands (Figure 1a,b). Every linker is coordinated to three SBUs, resulting in tetrahedral and adamantane-shaped cages with an internal pore diameter of respectively $4.8 \AA$ and $18 \AA$ (Figure 1c,d). These large pores are of interest for use of MOF- 808 as MPV reduction catalyst since they might alleviate mass transport limitations and facilitate formation of the six-membered transition state. Furthermore, a new crystalline form of MOF-808-designated as MOF-808-P-has been described; it contains five instead of six formate ligands per cluster, resulting in 
a coordinatively unsaturated Zr-site [32]. Citronellal cyclization experiments already indicated that MOF-808-P contains Lewis acid sites; such sites are suitable to catalyze MPV reduction [1].

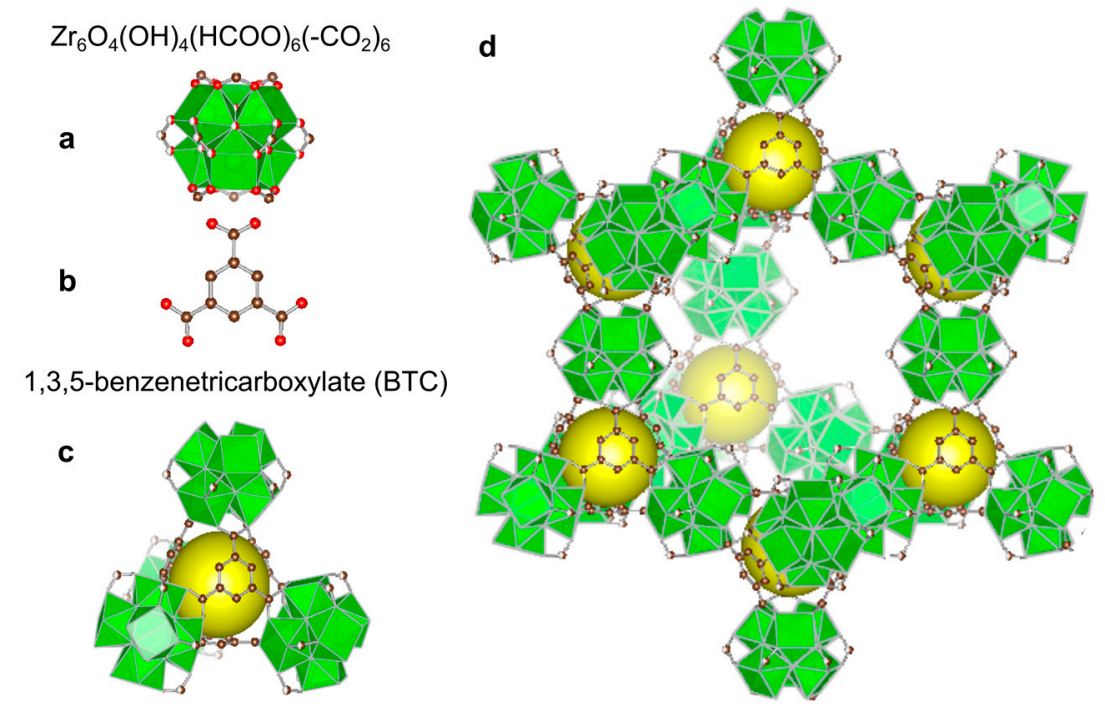

Figure 1. (a) $\mathrm{Zr}_{6} \mathrm{O}_{4}(\mathrm{OH})_{4}(\mathrm{HCOO})_{6}\left(-\mathrm{CO}_{2}\right)_{6}$ metal clusters, and (b) 1,3,5-benzenetricarboxylate (BTC) linkers form (c) tetrahedral cages (yellow spheres represent the internal free volume inside these cages with a diameter of $4.8 \AA$ ), and (d) large adamantane-shaped cages, internal free volume with diameter of $18 \AA$ A $\mathrm{Zr}$ coordination polyhedra, green; C: brown spheres; O: red spheres; formate $\mathrm{C}$ and O: half-filled; H omitted for clarity (based on Ref. [31]).

In this work, MOF-808-P is successfully used as MPV-catalyst for various $\alpha, \beta$-unsaturated carbonyl compounds. For cinnamaldehyde and citral, allylic alcohol yields of respectively $99 \%$ and $95 \%$ were obtained within a very short reaction time. More challenging substrates, such as $R$-carvone, reached the equilibrium point, but this equilibrium could be shifted towards the desired allyl alcohol by evaporation and the use of 1-Indanol.

\section{Results}

\subsection{Reduction of Cinnamaldehyde and Carvone}

Zr-based MOF-808-P was synthesized according to the reported procedure, starting from $\mathrm{ZrOCl}_{2} \cdot 8 \mathrm{H}_{2} \mathrm{O}$ and 1,3,5-benzenetricarboxylic acid (BTC) dissolved in a mixture of $\mathrm{N}, \mathrm{N}$-dimethylformamide (DMF) and formic acid [32]. In contrast to the MOF-808 synthesis employing stoichiometric amounts of linker $\left(\mathrm{ZrOCl}_{2} / \mathrm{BTC}=1 / 1\right)$ [31], MOF-808-P is synthesized with an excess of $\mathrm{Zr}$-source $\left(\mathrm{ZrOCl}_{2} / \mathrm{BTC}=3 / 1\right)$, resulting in formic acid ligand deficiency and an increased number of Lewis acid sites at the under-coordinated $\mathrm{Zr}$-metal. Characterization via powder X-ray diffraction (XRD, see supporting Figure S1), scanning electron microscopy (SEM, see supporting Figure S2) and thermogravimetric analysis (TGA, see supporting Figure S3) confirms that the correct structure is formed and that it crystallizes in octahedral microcrystals. Prior to the catalytic test, the metal-organic framework (MOF) is activated at $150{ }^{\circ} \mathrm{C}$ overnight to remove residual solvent molecules; at this temperature, activation under vacuum or in a regular oven had no effect on the resulting powder XRD nor on the catalytic activity.

Meerwein-Ponndorf-Verley (MPV) reductions were carried out at $120^{\circ} \mathrm{C}$ with isopropyl alcohol (IPA) as hydrogen donor and as solvent to move the equilibrium towards the desired allylic alcohol products. Catalytic activity of MOF-808-P is compared to UiO-66 and UiO-66- $\mathrm{NO}_{2}$ by keeping the $\mathrm{Zr}$ /substrate ratio constant ( $\mathrm{Zr}$ wt \% is based on inductively coupled plasma-atomic emission spectra (ICP-AES) measurements and theoretical structure calculations; see supporting 
information and Table S1). Figure 2 shows that MOF-808-P is an excellent MPV-catalyst with both high trans-cinnamaldehyde conversion $(>99 \%)$ and high trans-cinnamyl alcohol selectivity $(>99 \%)$. Not only the yield is higher compared to the UiO-66 materials; the reaction rate is considerably increased: after only $2 \mathrm{~h}$, complete conversion with high selectivity is obtained. The outstanding catalytic performance of MOF-808-P is even more clearly visible in the MPV reduction of $R$-carvone in Figure $2 \mathrm{~b}$. After $2 \mathrm{~h}$, the cis,trans-carveol yield is 20-fold higher than the yield obtained with $\mathrm{UiO}-66$ and $\mathrm{UiO}-66-\mathrm{NO}_{2}$ at the same time in the same reaction conditions. Even after reaction for $24 \mathrm{~h}$, the UiO- 66 materials only reach half of the MOF-808-P yield after $2 \mathrm{~h}$.

(a)
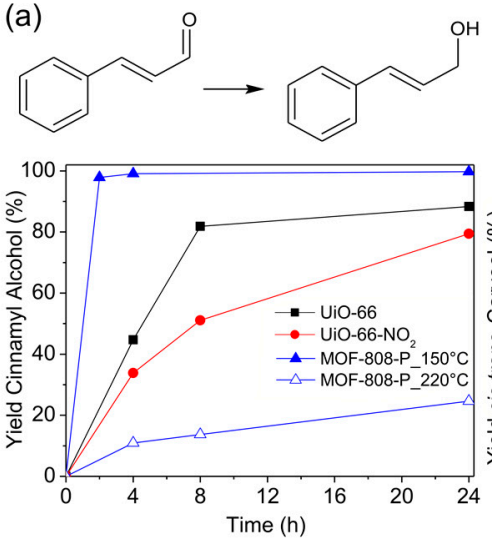

(b)
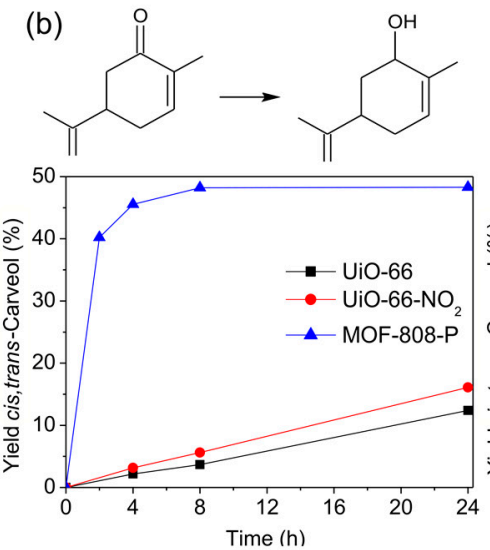

(c)
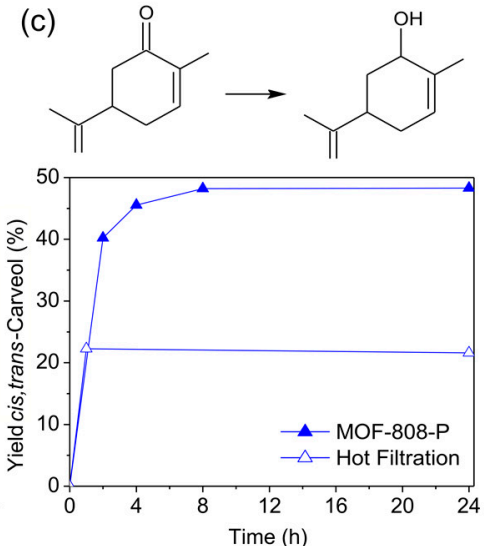

Figure 2. Meerwein-Ponndorf-Verley reduction of (a) trans-cinnamaldehyde and (b) $R$-carvone in isopropyl alcohol, catalyzed by Zr-based MOFs UiO-66- $x$ and MOF-808-P activated overnight at different temperatures: $150{ }^{\circ} \mathrm{C}(\boldsymbol{\Lambda})$ and $220^{\circ} \mathrm{C}(\Delta)$ (a: vacuum activation). (c) Hot filtration of the catalyst to confirm that the catalysis is truly heterogeneous. $\left(120^{\circ} \mathrm{C}, \mathrm{Zr} /\right.$ Substrate/IPA (Isopropyl alcohol) $=1 / 13 / 480,250 \mathrm{rpm}$ ).

The increased activity of MOF-808-P can be partly attributed to the larger pores, enhancing the accessibility of under-coordinated $\mathrm{Zr}$-atoms and facilitating the formation of the six-membered transition state, but more importantly to the Lewis acid sites on the under-coordinated $\mathrm{Zr}$-atoms. Similar to mechanisms reported in literature $[1,3,33]$, a metal-alkoxide bond is formed with an isopropyl alcohol molecule while the substrate carbonyl-group is simultaneously activated via similar coordination to a $\mathrm{Zr}$-atom. Both $\mathrm{C}-\mathrm{O}$ bonds in hydride donor and acceptor are in close proximity, and hydride transfer occurs via a six-membered transition state while maintaining stereochemistry. Probing of the Lewis acid sites via sorption with deuterated acetonitrile $\left(\mathrm{CD}_{3} \mathrm{CN}\right)$ (Figure S4) indicated a larger ratio of chemisorbed molecules on Lewis acid sites to physisorbed molecules (vibration frequency at $2297 \mathrm{~cm}^{-1}$ and $2265 \mathrm{~cm}^{-1}$, respectively) for MOF-808-P compared to UiO-66 after activation at $150{ }^{\circ} \mathrm{C}$. Furthermore, a large fraction of the $\mathrm{CD}_{3} \mathrm{CN}$ molecules on the Lewis acid sites of MOF-808-P stay chemisorbed upon desorption under vacuum by increasing temperature to $150{ }^{\circ} \mathrm{C}$, indicating a high acid strength.

It has often been observed that traces of DMF can be strongly adsorbed in the MOF structure, especially for MOF-808-P, since the missing formate ligand is most likely replaced by a DMF or water molecule [32]. ICP-AES indicates a $\mathrm{Zr}$ weight percentage of $39.4+/-0.7 \mathrm{wt} \%$, a little lower than the theoretical $40 \mathrm{wt} \%$ when all missing linkers would be replaced by water. To investigate if some residual DMF molecules might still be adsorbed in the MOF on the catalytically active sites as suggested by thermogravimetric analysis, the activation temperature was increased from $150{ }^{\circ} \mathrm{C}$ to $220^{\circ} \mathrm{C}$ under vacuum overnight. The elevated activation temperature clearly has a detrimental impact on the catalytic performance, as is shown in Figure 2a. Powder X-ray diffraction of MOF-808-P after activation at $220{ }^{\circ} \mathrm{C}$ indicates structure breakdown of the material after this activation step. 
Further experiments reported in this work were therefore always obtained with MOF-808-P catalyst activated at $150^{\circ} \mathrm{C}$.

Leaching of $\mathrm{Zr}$ into the solution was investigated by hot filtration of the reaction mixture after $1 \mathrm{~h}$ to remove the catalyst. Filtrate conversion did not increase after $24 \mathrm{~h}$, indicating that there is no leaching of catalytically-active $\mathrm{Zr}$ into the solution, and the catalysis is truly heterogeneous (Figure 2c). The stability of MOF-808-P was retained upon activation and during the course of the reaction, as indicated by powder X-ray diffraction (XRD) of recycled catalyst (supporting Figure S1). This recycled catalyst is collected via centrifugation after reaction and washed with ethanol and IPA four times, after which it was activated first at $60^{\circ} \mathrm{C}$ and overnight at $150^{\circ} \mathrm{C}$. Although hot filtration and powder XRD indicate there is no leaching nor perceptible structure breakdown, activity with the recycled catalyst drops by about $25 \%$ in the reduction of both cinnamaldehyde and carvone.

\subsection{Increase Equilibrium Carveol Yield}

In view of the remarkably short reaction time to reach the equilibrium point in the $R$-carvone reduction, this specific reaction is further investigated to increase the yield. As mentioned before, Meerwein-Ponndorf-Verley (MPV) reduction is an equilibrium reaction with the reverse reaction called Oppenauer oxidation. The Meerwein-Ponndorf-Verley-Oppenauer (MPVO) redox equilibrium is determined by the oxidation potentials of both carbonyl-alcohol pairs. Aside from using isopropyl alcohol as the solvent, two other strategies can be used to shift the equilibrium towards the desired products: (1) distilling off acetone and (2) the use of stronger reducing alcohols. The results of both strategies are shown in Figure 3.

(a)

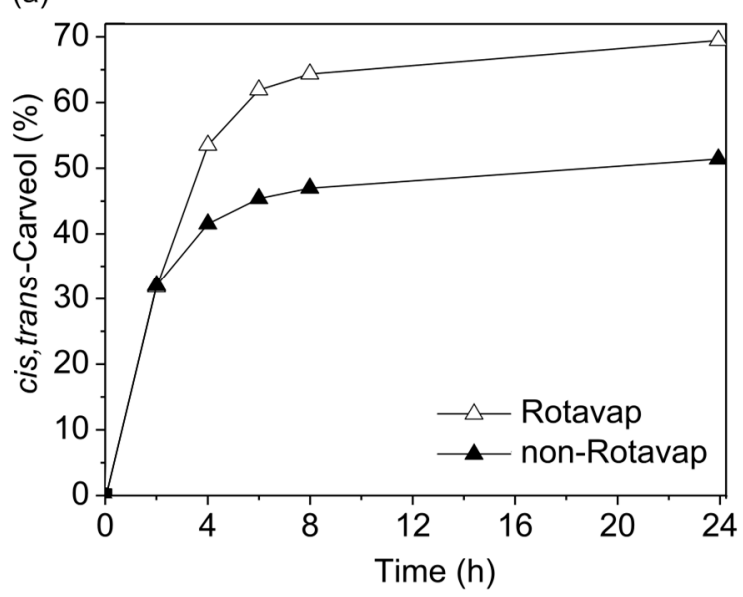

(b)

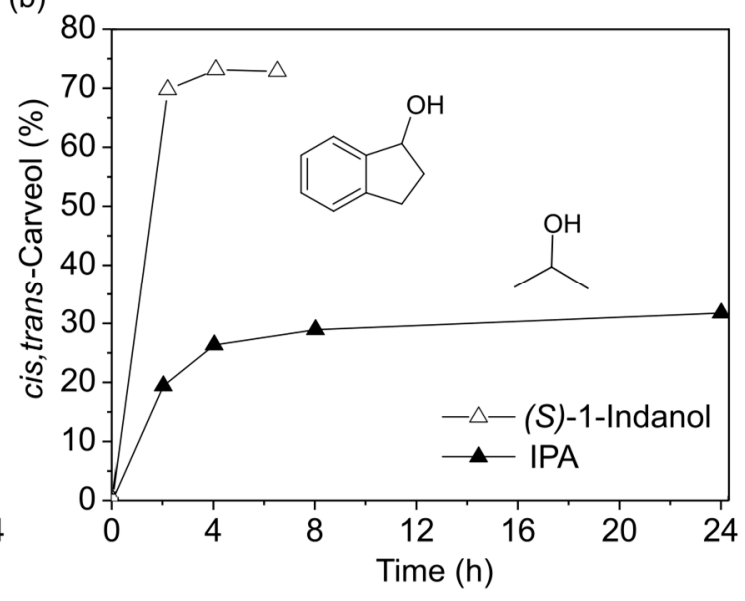

Figure 3. (a) Evaporation of acetone and isopropyl alcohol (IPA) and addition of fresh IPA solvent $(\Delta)$ direct the equilibrium towards a cis,trans-carveol yield of $70 \%\left(120^{\circ} \mathrm{C}, \mathrm{Zr} /\right.$ Substrate $\left./ \mathrm{IPA}=1 / 13 / 480\right)$. (b) MPV (Meerwein-Ponndorf-Verley) reduction with 12 equivalents of strongly reducing (S)-1-indanol $(\Delta)$ drives the equilibrium towards a cis,trans-carveol yield of $73 \%$. $\left(120^{\circ} \mathrm{C}, \mathrm{Zr} /\right.$ substrate/reducing agent $/$ toluene $=1 / 13 / 156 / 480)$.

\subsubsection{Evaporation of Acetone}

In the first strategy, two reactions are carried out simultaneously; one reaction is stopped after $2 \mathrm{~h}$ and both the solvent isopropyl alcohol and the formed acetone are removed by rotary vacuum evaporation. After the addition of fresh isopropyl alcohol, carveol yield reaches $70 \%$ (Figure 3a). This is $20 \%$ higher than without intermediate removal of acetone and clearly points out the importance of the reverse reaction, which limits the maximum carveol yield. Based on these results, the reaction was performed under reflux. Twenty mg of activated MOF-808-P together with IPA, internal standard, and carvone were placed in a $50 \mathrm{~mL}$ two-mounted flask connected to a condenser. The reaction mixture 
was refluxed at $80^{\circ} \mathrm{C}$ and stirred at $250 \mathrm{rpm}$, while a $\mathrm{N}_{2}$-flow was passed over the mixture to remove the formed acetone. The obtained yield after $4 \mathrm{~h}$ was comparable to the yield of the rotary evaporated sample (see supporting Figure S5). After 6 h, even 74\% yield could be obtained; however, loss of isopropyl alcohol was also significant at that time, so the reaction could no longer proceed.

\subsubsection{1-Indanol as Reducing Agent}

With the second strategy (the use of a stronger reducing alcohol $[7,24])$, the yield could be vastly increased to $73 \%$ (Figure $3 \mathrm{~b}$ ) with a diastereomeric excess (d.e.) for cis-carveol of $90 \%$ (see supporting information and supporting Figure S6). This reaction was carried out with 12 equivalents of $(S)-1$-indanol in toluene as solvent. The reaction with 12 equivalents of isopropyl alcohol in toluene is also given as comparison in Figure 3b.

\subsection{Substrate Scope}

Given the excellent catalytic performance of MOF-808-P both with trans-cinnamaldehyde and $R$-carvone, a series of other $\alpha, \beta$-unsaturated aldehydes and ketones were tested. Figure 4 shows that for the unsaturated aldehyde citral, high yields $(>90 \%)$ are also obtained within a short time with high selectivity ( $>98 \%)$. Furthermore, Figure 4 shows that for the $\alpha, \beta$-unsaturated ketones tested, complete conversion is not reached; instead, an equilibrium is established. This equilibrium is influenced by the redox potentials of the specific alcohol/ketone couples. We used isopropyl alcohol (IPA) as hydrogen donor and as solvent to shift the equilibrium towards the allylic alcohol product, but the oxidation potential of these compounds is too low compared to that of the acetone/IPA couple to allow complete conversion, as the reverse Oppenauer oxidation reaction takes place [6]. From the moment equilibrium is reached, selectivity starts to decrease due to the slow competing reactions that continue to progress. Selectivity in favor of the allylic alcohol is high in all reactions, only in the case of 4,4-dimethyl-2-cyclohexen-1-one, the saturated alcohol and etherification of the allylic alcohol with IPA are formed in significant amounts.

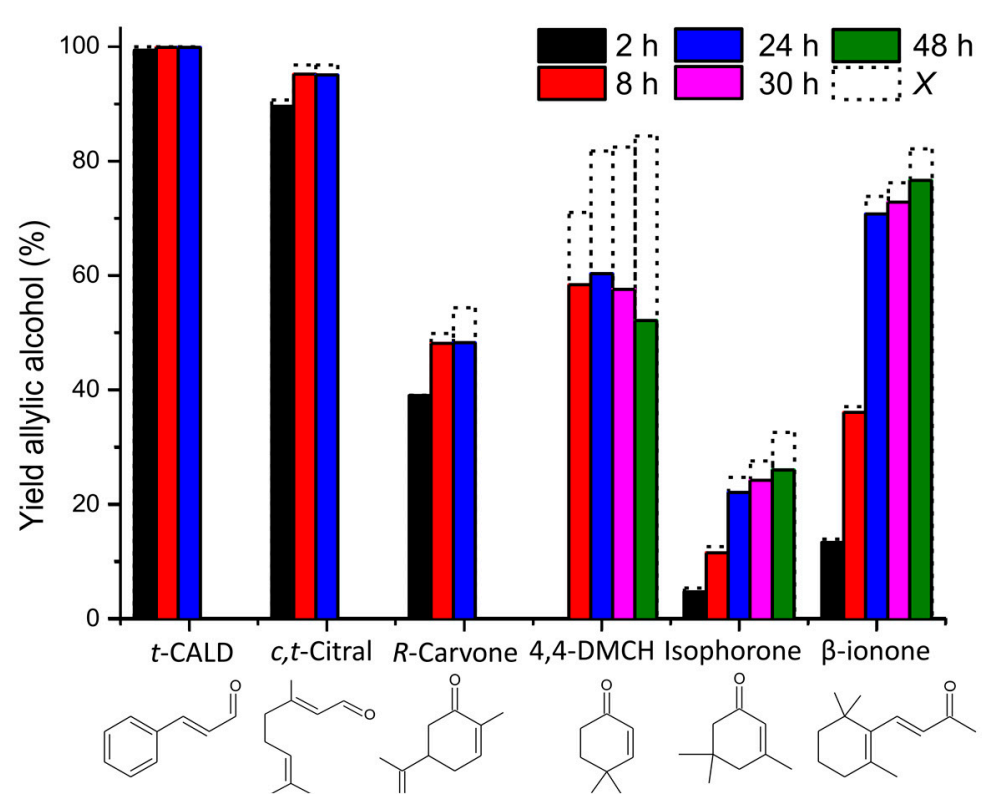

Figure 4. Meerwein-Ponndorf-Verley (MPV) reduction of $\alpha, \beta$-unsaturated carbonyl compounds catalyzed by MOF-808-P $\left(120^{\circ} \mathrm{C}, \mathrm{Zr} /\right.$ Substrate/IPA = 1/13/480, $\left.250 \mathrm{rpm}\right)$. Different colors indicate sampling at different reaction times; dotted bars represent conversion at the corresponding time $(X)$. 


\section{Discussion}

From the broad substrate scope shown in Figure 4, it can be concluded that the cages and channels in MOF-808-P are sufficiently large to allow chemoselective transfer hydrogenation of bulky molecules like $\beta$-ionone, with a yield of $77 \%$. Aside from steric effects, electronic effects are also known to have an impact on the reactivity [6,7]. Electron donating substituents (such as methyl groups) increase the electron density of the carbonyl group, thereby reducing the hydrogen transfer, especially when present in the $\beta$-position of the carbonyl group. This explains the relatively low yields for isophorone. Table 1 compares the allylic alcohol yields obtained with MOF-808-P, with the highest yields reported in literature for comparable catalyst systems. Compared to Zr-zeolite Beta, that uses $1.3 \mathrm{~mol} \% \mathrm{Zr}$ to reach full conversion after $3 \mathrm{~h}$, MOF-808-P obtained full cinnamaldehyde conversion within $2 \mathrm{~h}$ by using $7.7 \mathrm{~mol} \% \mathrm{Zr}$ (entry 1). For citral excellent allylic alcohol yield is also obtained within $4 \mathrm{~h}$, in contrast to the $\mathrm{Zr}$-PhyA reported MPV-catalyst that required $6 \mathrm{~h}$ to obtain $88 \%$ yield with a higher $\mathrm{Zr} \mathrm{mol} \mathrm{\%} \mathrm{(150 \% )} \mathrm{(entry} \mathrm{2).} \mathrm{The} \mathrm{reported} \mathrm{yield} \mathrm{for} \mathrm{carvone} \mathrm{and} \mathrm{isophorone} \mathrm{is} \mathrm{higher} \mathrm{than} \mathrm{the} \mathrm{one}$ obtained with MOF-808-P in our reaction system (entries 3 and 5); however, the reactant used in these studies (1-indanol) is more strongly reducing than the isopropanol used in this work. As mentioned above, the obtained carveol yield with indanol and MOF-808-P surpasses the best reported values (Table 1, entry 3). For comparison, a homogeneous $\mathrm{Zr}$-catalyst- $\mathrm{Zr}(\mathrm{IV})$ acetyl acetonate $\left(\mathrm{Zr}(\mathrm{acac})_{4}\right.$; obtained from ABCR, AB122355) - was also tested under the same reaction conditions as MOF-808-P in isopropyl alcohol with the same Zr-to-substrate ratio. After $2 \mathrm{~h}$ at $120^{\circ} \mathrm{C}$, very little conversion was measured; after $24 \mathrm{~h}$, however, $62 \%$ of the initial cinnamaldehyde was converted with $96 \%$ selectivity in favor of cinnamyl alcohol. This obtained cinnamyl alcohol yield with the homogeneous $\mathrm{Zr}$-catalysts is still 1.6 times lower than for MOF-808-P, while reaction time was 12 times longer. The same activity trend is observed in the MPV reduction of carvone; after $24 \mathrm{~h}$ at $120{ }^{\circ} \mathrm{C}$, conversion reaches only $7 \%$ with $\mathrm{Zr}(\mathrm{acac})_{4}$. These experiments indicate heterogenization of $\mathrm{Zr}$ in the form of a porous catalytically-active material is not only beneficial for its recyclability, but also for its activity.

Table 1. Comparison of MOF-808-P obtained allylic alcohol yields with literature.

\begin{tabular}{|c|c|c|c|c|c|c|c|}
\hline \multirow{2}{*}{ Entry } & \multirow{2}{*}{ Substrate } & \multirow{2}{*}{$t(\mathrm{~h})$} & \multirow{2}{*}{$Y^{1}(\%)$} & \multirow{2}{*}{$S^{1}(\%)$} & \multicolumn{3}{|c|}{ Literature $^{2}$} \\
\hline & & & & & Conditions & Catalyst & Ref. \\
\hline 1 & trans-cinnamaldehyde & 2 & 99 & 99 & $\begin{array}{c}96 \% 3 \mathrm{~h}, \\
\text { IPA }\end{array}$ & $\begin{array}{l}\text { Zr-zeolite } \beta \\
1.3 \text { mol \% Zr }\end{array}$ & [19] \\
\hline 2 & cis,trans-citral & 4 & 95 & 98 & $\begin{array}{l}88 \% 6 \mathrm{~h}, \\
\text { IPA }\end{array}$ & $\begin{array}{c}\text { Zr-PhyA } \\
150 \text { mol \% Zr }\end{array}$ & [15] \\
\hline 3 & $R$-carvone & 8 & 48 & 97 & $\begin{array}{c}62 \% 5 \mathrm{~h}, \\
1 \text {-indanol }\end{array}$ & $\begin{array}{l}\text { Zr-MCM-41 } \\
5 \mathrm{~mol} \% \mathrm{Zr}\end{array}$ & [7] \\
\hline 4 & $\begin{array}{l}\text { 4,4-dimethyl-2- } \\
\text { cyclohexen-1- } \\
\text { one }\end{array}$ & 24 & 60 & 85 & - & - & - \\
\hline
\end{tabular}


Table 1. Cont.

\begin{tabular}{|c|c|c|c|c|c|c|c|}
\hline \multirow{2}{*}{ Entry } & \multirow{2}{*}{ Substrate } & \multirow{2}{*}{$t(\mathrm{~h})$} & \multirow{2}{*}{$Y^{1}(\%)$} & \multirow{2}{*}{$S^{1}(\%)$} & \multicolumn{3}{|c|}{ Literature $^{2}$} \\
\hline & & & & & Conditions & Catalyst & Ref. \\
\hline 5 & isophorone & 48 & 24 & 75 & $\begin{array}{l}40 \% 24 \mathrm{~h}, \\
\text { 1-indanol }\end{array}$ & $\begin{array}{l}\text { Hf-MCM-41 } \\
5 \mathrm{~mol} \% \mathrm{Hf}\end{array}$ & [24] \\
\hline 6 & $\beta$-ionone & 48 & 77 & 89 & $\begin{array}{c}77 \% \\
9 \text { h, IPA }\end{array}$ & $\begin{array}{l}\text { B-MCM-41 } \\
4 \mathrm{~mol} \% \text { B }\end{array}$ & [23] \\
\hline
\end{tabular}

\section{Materials and Methods}

\subsection{Synthesis and Characterization}

All chemicals and solvents used in the syntheses were of reagent grade and used without further purification.

\subsubsection{MOF Synthesis}

The synthesis was performed according to the reported procedure. All materials were made in a closed Schott DURAN $®(V W R$, Leuven, Belgium) pressure plus bottle with a volume of $1 \mathrm{~L}$ under static conditions starting from a non-equimolar solution of $\mathrm{ZrOCl}_{2} \cdot 8 \mathrm{H}_{2} \mathrm{O}(9.7 \mathrm{~g}, 30 \mathrm{mmol}, 95 \%, \mathrm{ABCR}$ $\mathrm{GmbH}$, Karlsruhe, Germany) and 1,3,5-benzenetricarboxylic acid (BTC) $(2.1 \mathrm{~g}, 10 \mathrm{mmol}, 98 \%$, ABCR $\mathrm{GmbH}$, Karlsruhe, Germany) dissolved in a mixture of $\mathrm{N}, \mathrm{N}$-dimethylformamide (DMF) (450 mL, $6 \mathrm{~mol}$, $>99 \%$, Acros Organics, Geel, Belgium) and formic acid (450 mL, 12 mol, >98\%, Acros Organics, Geel, Belgium). The synthesis mixture was placed in a preheated oven at $120^{\circ} \mathrm{C}$ for $48 \mathrm{~h}$. The powder was collected via centrifugation (10 min, $11000 \mathrm{rpm}$ ) and thoroughly washed with DMF (3 times/day for 3 days) and methanol (HPLC grade, Chem-Lab Analytical BVBA, Zedelgem, Belgium) (3 times/day for 3 days). Finally, the material was activated at $150{ }^{\circ} \mathrm{C}$ in air to remove residual solvent molecules.

\subsubsection{MOF Characterization}

Powder X-ray diffractograms were routinely collected on a STOE STADI COMBI P diffractometer (STOE \& Cie GmbH, Darmstadt, Germany) in High-Throughput mode, equipped with an image plate detector using $\mathrm{Cu} \mathrm{K} \alpha$ radiation $(\lambda=1.54056 \AA)$. Scanning Electron Microscopy (SEM) images were obtained using a JEOL SEM (JSM-6010LV, Jeol Europe BV, Zaventem, Belgium). MOF-808-P was analyzed by Thermogravimetric Analysis (TGA) under a stream of $\mathrm{O}_{2}$-gas using a Universal V4.5A TA Instrument (TA Instruments, New Castle, DE, USA). $\mathrm{CD}_{3} \mathrm{CN}$ chemisorption spectra were measured on a Nicolett 6700 (Thermo Scientific, Waltham, MA, USA) FTIR (128 scans, resolution of $2 \mathrm{~cm}^{-1}$ ) and analyzed with Omnic software (Version 8.0, Thermo Scientific). Inductively Coupled Plasma (ICP)—Atomic Emission Spectra (AES) were recorded on a Varian 720-ES (Varian Inc., Walnut Creek, CA, USA).

\subsection{Catalytic Experiments}

Before reaction, each catalyst was dried at $150{ }^{\circ} \mathrm{C}$ for $16 \mathrm{~h}$ to remove residual solvent molecules. Catalytic reactions were carried out in $10 \mathrm{~mL}$ glass crimp cap vials loaded with 20-30 mg catalyst and a magnetic stirring bar. A solution of the substrate in $3.3 \mathrm{~mL}$ isopropyl alcohol (IPA) (extra pure, Acros Organics, Geel, Belgium) was added; tetradecane (99\%, TCI Europe NV, Zwijndrecht, Belgium) was added as internal standard. For each catalyst and substrate, a substrate-to-Zr ratio of 7.8 was used to 
compare the activity of each catalyst for every substrate. After introduction of the reaction mixture, the vials were placed in an aluminum heating block (at $\left.120^{\circ} \mathrm{C}\right)$ and stirred $(250 \mathrm{rpm})$. Reaction samples were filtered through a $0.45 \mu \mathrm{m}$ PTFE filter (Thermo scientific, VWR, Leuven, Belgium) and analyzed with a Shimadzu 2010 GC (Shimadzu Benelux BV, Brussel, Belgium) equipped with a CP-Sil 8 column and a FID detector. Reaction products were identified using GC-MS (Agilent Technologies Belgium, Diegem, Belgium).

\section{Conclusions}

The Zr-based MOF-808-P was successfully used as a Meerwein-Ponndorf-Verley reduction catalyst for various $\alpha, \beta$-unsaturated aldehydes and ketones. For typical substrates such as trans-cinnamaldehyde and cis,trans-citral, yields of respectively $99 \%$ and $95 \%$ were obtained within a short reaction time. Because of the increased number and strength of Lewis acid sites on coordinatively unsaturated $\mathrm{Zr}$-atoms, the cis,trans-carveol yield increased 20-fold compared to reactions using another $\mathrm{Zr}$-based MOF, UiO-66. For more challenging substrates, the equilibrium and reverse reduction of acetone prevented complete conversion. Two strategies were successfully used to shift the equilibrium towards the desired allylic alcohol products: evaporation of formed acetone and the use of the more strongly reducing 1-indanol increased the cis,trans-carveol yield from $50 \%$ to more than $70 \%$.

Supplementary Materials: The following are available online at www.mdpi.com/2073-4344/6/7/104/s1, Figure S1: Powder X-ray diffractograms of MOF-808-P: simulated MOF-808 pattern, as synthesized MOF-808-P, activated at $150{ }^{\circ} \mathrm{C}$ and $200{ }^{\circ} \mathrm{C}$ for $16 \mathrm{~h}$ and recycled after reaction. Figure S2: SEM micrograph of MOF-808-P. Figure S3: Thermogravimetric Analysis. Figure S4: Normalized FTIR spectra of $\mathrm{CD}_{3} \mathrm{CN}$ chemisorption on MOF-808-P (a) and UiO-66 (b) at 10 mbar (RT) and desorption under vacuum at increasing temperature. Figure S5: Evaporation of acetone and isopropanol and addition of fresh IPA solvent ( $\square$ ) indicate that the equilibrium of the Meerwein-Ponndorf-Verley (MPV) reduction of $R$-carvone with MOF-808-P can be shifted towards cis,trans-carveol. By performing the reaction under reflux at 80 to $100{ }^{\circ} \mathrm{C}$, yield could be increased to $74 \%$. Figure S6: MPV reduction of $R$-carvone with MOF-808-P with 5 (filled) or 12 (open) equivalents of (S)-1-Indanol. When yield of cis,trans-carveol $(\boldsymbol{\square}, \square)$ reaches equilibrium $(73 \%)$, epimerization occurs and the diastereomeric excess (d.e., $\bullet, \bigcirc)$ decreases. $\left(120^{\circ} \mathrm{C}, \mathrm{Zr} /\right.$ substrate/reducing agent/toluene $\left.=1 / 13 / 156 / 480\right)$. Table S1: Theoretical molecular formula and Zr-percentage compared to ICP-AES analysis of self-synthesized MOF-808-P activated at $150{ }^{\circ} \mathrm{C}(16 \mathrm{~h})$.

Acknowledgments: The authors thank the Research Foundation-Flanders (FWO) (PhD scholarship to E.P.), the China Scholarship Council (PhD scholarship to G.F.), the European Union (Horizon 2020) and the Marie Sklodowska-Curie innovation program (Grant 641887 for PhD scholarship to C.Y.X.T.) and Belspo (IAP-VII/05) for financial support.

Author Contributions: M.B.J.R. and D.E.D.V. conceived the project; E.P. performed the catalytic experiments, analyzed the data and wrote the paper; G.F. synthesized and characterized the materials via SEM and TGA; C.Y.X.T. performed $\mathrm{CD}_{3} \mathrm{CN}$ chemisorption measurements.

Conflicts of Interest: The authors declare no conflict of interest.

\section{Abbreviations}

The following abbreviations are used in this manuscript:

$\begin{array}{ll}\text { BTC } & \text { 1.3.5-benzenetricarboxylic acid } \\ \text { IPA } & \text { Isopropyl alcohol } \\ \text { MOF } & \text { Metal-Organic Framework } \\ \text { MPV } & \text { Meerwein-Ponndorf-Verley } \\ \text { XRD } & \text { X-ray diffraction }\end{array}$

\section{References}

1. Sheldon, R.A.; van Bekkum, H. Fine Chemicals through Heterogeneous Catalysis; Wiley-VCH: Weinheim, Germany, 2008.

2. Chuah, G.K.; Jaenicke, S.; Zhu, Y.Z.; Liu, S.H. Meerwein-Ponndorf-Verley reduction over Heterogeneous Catalysts. Curr. Org. Chem. 2006, 10, 1639-1654. [CrossRef]

3. Liu, S.H.; Jaenicke, S.; Chuah, G.K. Hydrous Zirconia as a Selective Catalyst for the Meerwein-Ponndorf-Verley Reduction of Cinnamaldehyde. J. Catal. 2002, 206, 321-330. [CrossRef] 
4. Gladiali, S.; Alberico, E. Asymmetric transfer hydrogenation: Chiral ligands and applications. Chem. Soc. Rev. 2006, 35, 226-236. [CrossRef] [PubMed]

5. Boronat, M.; Corma, A.; Renz, M. Mechanism of the Meerwein-Ponndorf-Verley-Oppenauer (MPVO) Redox Equilibrium on Sn- and Zr-Beta Zeolite Catalysts. J. Phys. Chem. B 2006, 110, 21168-21174. [CrossRef] [PubMed]

6. Noyori, R.; Hashiguchi, S. Asymmetric Transfer Hydrogenation Catalyzed by Chiral Ruthenium Complexes. Acc. Chem. Res. 1997, 30, 97-102. [CrossRef]

7. De bruyn, M.; De Vos, D.E.; Jacobs, P.A. Chemoselective Hydrogen Transfer Reduction of Unsaturated Ketones to Allylic Alcohols with Solid Zr and Hf Catalysts. Adv. Synth. Catal. 2002, 344, 1120-1125. [CrossRef]

8. Wilds, A.L. Reduction with Aluminum Alkoxides. Org. React. 1944, 2, 178-223.

9. McNerney, B.; Whittlesey, B.; Cordes, D.B.; Krempner, C. A well-defined monomeric aluminum complex as an efficient and general catalyst in the Meerwein-Ponndorf-Verley reduction. Chem.-A Eur. J. 2014, 20, 14959-14964. [CrossRef] [PubMed]

10. Nandi, P.; Solovyov, A.; Okrut, A.; Katz, A. Al ${ }^{\mathrm{III}}$-Calix[4]arene Catalysts for Asymmetric-Meerwein-Ponndorf-Verley Reduction. ACS Catal. 2014, 4, 2492-2495. [CrossRef]

11. Nandi, P.; Matvieiev, Y.I.; Boyko, V.I.; Durkin, K.A.; Kalchenko, V.I.; Katz, A. MPV reduction using $\mathrm{Al}^{\mathrm{III}}$-calix[4]arene Lewis acid catalysts: Molecular-level insight into effect of ketone binding. J. Catal. 2011, 284, 42-49. [CrossRef]

12. Zhu, Y.; Liu, S.; Jaenicke, S.; Chuah, G. Zirconia catalysts in Meerwein-Ponndorf-Verley reduction of citral. Catal. Today 2004, 97, 249-255. [CrossRef]

13. Ramanathan, A.; Castro Villalobos, M.C.; Kwakernaak, C.; Telalovic, S.; Hanefeld, U. Zr-TUD-1: A Lewis Acidic, three-dimensional, mesoporous, zirconium-containing catalyst. Chem.-A Eur. J. 2008, 14, 961-972. [CrossRef] [PubMed]

14. Vermoortele, F.; Bueken, B.; Le Bars, G.; Van de Voorde, B.; Vandichel, M.; Houthoofd, K.; Vimont, A.; Daturi, M.; Waroquier, M.; Van Speybroeck, V.; et al. Synthesis Modulation as a Tool To Increase the Catalytic Activity of Metal-Organic Frameworks: The Unique Case of UiO-66(Zr). J. Am. Chem. Soc. 2013, 135, 11465-11468. [CrossRef] [PubMed]

15. Song, J.; Zhou, B.; Zhou, H.; Wu, L.; Meng, Q.; Liu, Z.; Han, B. Porous Zirconium-Phytic Acid Hybrid: A Highly Efficient Catalyst for Meerwein-Ponndorf-Verley Reductions. Angew. Chemie Int. Ed. 2015, 127, 9531-9535. [CrossRef]

16. Ivanov, V.A.; Bachelier, J.; Audry, F.; Lavalley, J.C. Study of the Meerwein-Pondorff-Verley reaction between ethanol and acetone on various metal oxides. J. Mol. Catal. 1994, 91, 45-59. [CrossRef]

17. Battilocchio, C.; Hawkins, J.M.; Ley, S.V. A Mild and Efficient Flow Procedure for the Transfer Hydrogenation of Ketones and Aldehydes using Hydrous Zirconia. Org. Lett. 2013, 15, 2278-2281. [CrossRef] [PubMed]

18. Zhu, Y.; Jaenicke, S.; Chuah, G.K. Supported zirconium propoxide-A versatile heterogeneous catalyst for the Meerwein-Ponndorf-Verley reduction. J. Catal. 2003, 218, 396-404. [CrossRef]

19. Zhu, Y.; Chuah, G.; Jaenicke, S. Selective Meerwein-Ponndorf-Verley reduction of $\alpha$, $\beta$-unsaturated aldehydes over Zr-zeolite beta. J. Catal. 2006, 241, 25-33. [CrossRef]

20. Iglesias, J.; Melero, J.A.; Morales, G.; Moreno, J.; Segura, Y.; Paniagua, M.; Cambra, A.; Hernández, B. Zr-SBA-15 Lewis Acid Catalyst: Activity in Meerwein Ponndorf Verley Reduction. Catalysts 2015, 5, 1911-1927. [CrossRef]

21. Wang, J.; Okumura, K.; Jaenicke, S.; Chuah, G.-K. Post-synthesized zirconium-containing Beta zeolite in Meerwein-Ponndorf-Verley reduction: Pros and cons. Appl. Catal. A Gen. 2015, 493, 112-120. [CrossRef]

22. Yuan, Y.; Yao, S.; Wang, M.; Lou, S.; Yan, N. Recent Progress in Chemoselective Hydrogenation of $\alpha, \beta$-Unsaturated Aldehyde to Unsaturated Alcohol Over Nanomaterials. Curr. Org. Chem. 2013, 17, 400-413. [CrossRef]

23. Uysal, B.; Oksal, B.S. Comparison of heterogeneous $\mathrm{B}\left(\mathrm{O}^{i} \mathrm{Pr}\right)_{3}-\mathrm{MCM}-41$ and homogeneous $\mathrm{B}\left(\mathrm{O}^{i} \mathrm{Pr}\right)_{3}, \mathrm{~B}(\mathrm{OEt})_{3}$ catalysts for chemoselective MPV reductions of unsaturated aldehydes and ketones. Appl. Catal. A Gen. 2012, 435-436, 204-216. [CrossRef]

24. De bruyn, M.; Limbourg, M.; Denayer, J.; Baron, G.V.; Parvulescu, V.; Grobet, P.J.; De Vos, D.E.; Jacobs, P.A. Mesoporous $\mathrm{Zr}$ and $\mathrm{Hf}$ catalysts for chemoselective MPV reductions of unsaturated ketones. Appl. Catal. A Gen. 2003, 254, 189-201. [CrossRef] 
25. Gascon, J.; Corma, A.; Kapteijn, F.; Llabre, F.X. Metal Organic Framework Catalysis: Quo vadis? ACS Catal. 2014, 4, 361-378. [CrossRef]

26. Dhakshinamoorthy, A.; Opanasenko, M.; Čejka, J.; Garcia, H. Metal organic frameworks as heterogeneous catalysts for the production of fine chemicals. Catal. Sci. Technol. 2013, 3, 2509-2540. [CrossRef]

27. Dhakshinamoorthy, A.; Asiri, A.M.; Garcia, H. Catalysis by metal-organic frameworks in water. Chem. Commun. 2014, 50, 12800-12814. [CrossRef] [PubMed]

28. Plessers, E.; De Vos, D.E.; Roeffaers, M.B.J. Chemoselective reduction of $\alpha, \beta$-unsaturated carbonyl compounds with UiO-66 materials. J. Catal. 2016, 340, 136-143. [CrossRef]

29. Cirujano, F.G.; Corma, A.; i Xamena, F.X.L. Conversion of levulinic acid into chemicals: Synthesis of biomass derived levulinate esters over Zr-containing MOFs. Chem. Eng. Sci. 2015, 124, 52-60. [CrossRef]

30. Valenzano, L.; Civalleri, B.; Chavan, S.; Bordiga, S.; Nilsen, M.H.; Jakobsen, S.; Lillerud, K.P.; Lamberti, C. Disclosing the Complex Structure of UiO-66 Metal Organic Framework: A Synergic Combination of Experiment and Theory. Chem. Mater. 2011, 23, 1700-1718. [CrossRef]

31. Furukawa, H.; Gándara, F.; Zhang, Y.; Jiang, J.; Queen, W.L.; Hudson, M.R.; Yaghi, O.M. Water Adsorption in Porous Metal-Organic Frameworks and Related Materials. J. Am. Chem. Soc. 2014, 136, 4369-4381. [CrossRef] [PubMed]

32. Jiang, J.; Gándara, F.; Zhang, Y.; Na, K.; Yaghi, O.M. Superacidity in sulfated metal-organic framework-808. J. Am. Chem. Soc. 2014, 136, 12844-12847. [CrossRef] [PubMed]

33. Creyghton, E.J.; Ganeshie, S.D.; Downing, R.S.; van Bekkum, H. Stereoselective Meerwein-Ponndorf-Verley and Oppenauer reactions catalysed by zeolite BEA. J. Mol. Catal. A Chem. 1997, 115, 457-472. [CrossRef]

(C) 2016 by the authors; licensee MDPI, Basel, Switzerland. This article is an open access article distributed under the terms and conditions of the Creative Commons Attribution (CC-BY) license (http:/ / creativecommons.org/licenses/by/4.0/). 